\title{
MSSM baryogenesis and electric dipole moments: an update on the phenomenology
}

\author{
V. Cirigliano, ${ }^{a}$ Yingchuan Li, $^{b}$ S. Profumo ${ }^{c}$ and M.J. Ramsey-Musolf ${ }^{b, d}$ \\ a Theoretical Division, Los Alamos National Laboratory, \\ Los Alamos NM 87545, U.S.A. \\ ${ }^{b}$ Department of Physics, University of Wisconsin, \\ 1150 University Avenue, Madison, WI 53705, U.S.A. \\ ${ }^{c}$ Department of Physics and Santa Cruz Institute for Particle Physics, University of California, \\ 1156 High St., Santa Cruz, CA 95064, U.S.A. \\ ${ }^{d}$ Kellogg Radiation Laboratory, California Institute of Technology, \\ 1200 E California Blvd, Pasadena, CA 91125 U.S.A. \\ E-mail: cirigliano@lanl.gov, yli@physics.wisc.edu, \\ profumo@scipp.ucsc.edu, mjrm@physics.wisc.edu
}

ABSTRACT: We explore the implications of electroweak baryogenesis for future searches for permanent electric dipole moments in the context of the minimal supersymmetric extension of the Standard Model (MSSM). From a cosmological standpoint, we point out that regions of parameter space that over-produce relic lightest supersymmetric particles can be salvaged only by assuming a dilution of the particle relic density that makes it compatible with the dark matter density: this dilution must occur after dark matter freeze-out, which ordinarily takes place after electroweak baryogenesis, implying the same degree of dilution for the generated baryon number density as well. We expand on previous studies on the viable MSSM regions for baryogenesis, exploring for the first time an orthogonal slice of the relevant parameter space, namely the $\left(\tan \beta, m_{A}\right)$ plane, and the case of non-universal relative gaugino-higgsino $\mathrm{CP}$ violating phases. The main result of our study is that in all cases lower limits on the size of the electric dipole moments exist, and are typically on the same order, or above, the expected sensitivity of the next generation of experimental searches, implying that MSSM electroweak baryogenesis will be soon conclusively tested.

KEYWORDS: Supersymmetry Phenomenology 


\section{Contents}

1 Introduction 1

2 MSSM baryogenesis and EDMs 4

$\begin{array}{lll}3 & \text { Baryogenesis and neutralino relic (over-)abundance } & 7\end{array}$

4 A lower limit on the electron and neutron EDM 10

$5 \quad$ EDM in the bino-driven electroweak baryogenesis scenario 12

6 The $\left(\tan \beta, m_{A}\right)$ plane $\quad 13$

$\begin{array}{lll}7 & \text { Discussion and conclusions } & 19\end{array}$

\section{Introduction}

Explaining the origin of the baryonic matter of the Universe remains an open problem at the interface of cosmology with particle and nuclear physics. Consistent values for the ratio $Y_{B}$ of the baryon number density $\left(n_{B}\right)$ to entropy density $(s)$ have been obtained from measured light element abundances in the context of Big Bang Nucleosynthesis (BBN) [1] and acoustic oscillations in the cosmic microwave background (CMB) as measured by the Wilkinson Microwave Anisotropy Probe (WMAP) [1, 2]:

$$
Y_{B}=n_{B} / s=\left\{\begin{array}{cc}
(6.7-9.2) \times 10^{-11} & \mathrm{BBN} \\
(8.36-9.32) \times 10^{-11} & \mathrm{CMB}
\end{array}\right.
$$

Assuming that the $Y_{B}=0$ at the end of the inflationary epoch, the dynamics of the subsequently evolving cosmos would then have generated a non-vanishing baryon asymmetry. As first noted by Sakharov, [3], these dynamics must have included violation of baryon number (B) conservation; violation of $\mathrm{C}$ and $\mathrm{CP}$ conservation; and a departure from equilibrium dynamics assuming CPT invariance.

Although it is not known when in the history of the universe these ingredients came into play, one possibility that can be tested with terrestrial experiments is that baryogenesis occurred during the era of electroweak symmetry breaking. In this scenario, which we consider here, a departure from equilibrium occurs through a strong, first order electroweak phase transition (EWPT) in which bubbles of broken electroweak symmetry form in the symmetric background. Charge asymmetries generated by CP-violating interactions at the bubble walls diffuse into the unbroken phase, where B-violating electroweak sphalerons convert them into non-vanishing baryon number density. The expanding bubbles capture 
this baryon number density by quenching the sphaleron transitions, ultimately leading to a relic baryon asymmetry at zero temperature.

In principle, the Standard Model (SM) of particle physics could have satisfied these "Sakharov criteria" as needed for electroweak baryogenesis, particularly as the B-violating sphaleron transitions associated with the $\mathrm{SU}(2)_{L}$ gauge sector are unsuppressed at temperatures of order $100 \mathrm{GeV}$ and above. However, the strength of the SM CP-violating (CPV) interactions are too small to have generated sufficiently large charge asymmetries needed to bias the sphalerons into making baryons. In addition, the lower bound on the mass of the SM Higgs boson [4] implies that finite temperature SM effective scalar potential that governs electroweak symmetry-breaking would not admit a sufficiently strong first order EWPT as needed to prevent a "washout" of any baryon asymmetry that might otherwise have been produced at high temperatures $[5,6]$. Consequently, an explanation of the observed $Y_{B}$ requires new physics beyond the Standard Model .

In this paper, we concentrate on the possibility that this new physics involved $\mathrm{TeV}$ scale supersymmetric interactions in the guise of the minimal supersymmetric Standard Model (MSSM). The general motivation for considering TeV scale supersymmetry is well-known, and we refer the reader to the vast literature for a discussion [7]. Here, we focus on the minimal scenario, up-dating our previous study to take into account several developments (outlined below). The phenomenological constraints on MSSM electroweak baryogenesis (EWB) are quite stringent: the existence of a strong first order EWPT requires both a relatively light SM-like Higgs scalar and a correspondingly light scalar superpartner of the right-handed top quark. Searches for scalars at the Large Hadron Collider (LHC) will readily explore the narrow window of available parameter space for these considerations. The strengths of MSSM CPV interactions relevant for EWB are similarly tightly bounded by current limits on the permanent electric dipole moments (EDMs) of the electron, neutron, and mercury atom, with bounds on the latter having recently improved by a factor of seven [8]. Thus, it is conceivable that the combination of LHC studies and planned EDM searches having substantially improved sensitivities may rule out the minimal version of supersymmetric EWB, leaving other scenarios such as non-minimal supersymmetry or leptogenesis as the most viable possibilities. The purpose of the present study is to delineate just how close we are to such a situation.

The new recent developments in supersymmetric EWB include the following results:

(i) Carena et al. [9] have completed an analysis of the EWPT in the MSSM using a twoloop, finite temperature effective potential computed with effective theory to integrate out all but the most relevant scalar degrees of freedom. Their results indicate that a sufficiently strong first order EWPT can occur if the SM-like Higgs and RH stop masses are less than $125 \mathrm{GeV}$, with some dependence on the cutoff scale of the effective theory. For the EWPT-viable regions of parameter space, the electroweak minimum is metastable with a lifetime longer than the age of the universe. The presence of the light, $\mathrm{RH}$-stop leads to the existence of a deeper zero-temperature color-breaking minimum that can be avoided if the electroweak minimum is deeper at temperatures associated with the EWPT. 
(ii) Three of us have recently completed a two-loop computation of the electric dipole moments of the electron and neutron in the MSSM, including all of the CPV interactions that are most likely to be responsible for EWB in the MSSM [10]. The results indicate that even under the most optimistic scenarios, viable MSSM EWB requires that the CPV phases in the gaugino-Higgs-Higgsino sector be non-universal, in contrast to the usual universality assumptions. The CPV interaction most likely to lead to successful EWB involves the bino and Higgsino fermions [11].

(iii) It was shown in ref. $[12,13]$ that the value of $\tan \beta$ could play a decisive role in the transport dynamics that convert the bino-driven Higgsino asymmetry into a lefthanded SM fermion number asymmety - the quantity that ultimately biases the sphalerons into making baryons. Larger values of $\tan \beta$ lead to a suppression of $Y_{B}$ and a potentially significant dependence on the masses of the RH bottom squarks $\left(\tilde{b}_{R}\right)$ and tau sleptons $\left(\tilde{\tau}_{R}\right)$, whose Yukawa couplings are enhanced with increasing $\tan \beta$. For values of this parameter favored by the muon anomalous magnetic moment, one expects a suppression of a factor of two or more - and possibly even a sign change in $Y_{B}$ - depending on the $\mathrm{RH}$ sbottom and stau masses.

In addition to analyzing the phenomenological implications of some of these developments for MSSM EWB and EDM searches, we also take into account another physical consideration not treated in previous work, namely the impact of the cold dark matter (CDM) relic density at the time the baryons are produced. In some regions of parameter space in which MSSM EWB is naïvely viable, one expects $\Omega_{\mathrm{CDM}}$ due to neutralinos to be larger than the observed relic density. In standard cosmological scenarios, these parameter space regions are therefore excluded. They may become viable in non-standard scenarios involving late-time reheating that lead to a dilution of the neutralino density due to entropy production. This entropy dilution, however, will also lead to a reduction in $Y_{B}$. We find that the resulting impact on the EWB-allowed MSSM parameter space can be substantial, particularly in regions where wino-Higgsino driven EWB might otherwise have been potentially important. The impact on the bino-Higgsino driven scenario is less pronounced since in this region of parameter space the neutralino relic density typically lies below the observed value so that no entropy dilution rescaling occurs.

Taking all of the aforementioned considerations into account, we find minimum values for $\left|d_{e}\right|$ and $\left|d_{n}\right|$ for which MSSM EWB would be viable under the most optimistic scenarios. These values lie somewhat below the expected sensitivities of the next generation of EDM searches, though given outstanding theoretical uncertainties (discussed below) we cannot make a definitive statement until further theoretical progress is achieved. For the interim, we provide this analysis as a phenomenological "progress report" that will undoubtedly require further updates in the future. To this end, we illustrate the key features of the current status in several figures:

(a) a series (figure 1-6) that shows the EWB-viable regions in the plane of gauginoHiggsino mass parameters for different values of the CP-violating phases and corresponding values of the EDMs. In this series, we show the impact of imposing the 
requirements of entropy dilution needed for consistency with the observed CDM relic density;

(b) a series of plots (figure 7-10) in the space of $\tan \beta$ and the mass of the CP-odd scalar, $m_{A}$.

We discuss in section 2 the general framework, and we outline the MSSM parameter space compatible with EWB. In this section only we consider a light right handed stop as the driver of a strongly first order EW phase transition: in the rest of the paper we drop this assumption, and always consider

(i) a heavy sfermion sector (including both stops) and

(ii) beyond the MSSM physics driving a strongly first order EWPT. In section 3 we discuss the relation between the neutralino relic abundance and EWB.

For purposes of comparison with our earlier work, we consider here a scenario of universal CPV gaugino-Higgsino-Higgs phases without imposing the EDM constraints. The latter are treated in sections 4 and 5 . Constraints in the $\tan \beta-m_{A}$ plane are given in section 6 . We summarize our findings and the outstanding theoretical issues in section 7 .

\section{MSSM baryogenesis and EDMs}

For purposes of our analysis, in this section (and only here) we start with the customary assumption that the RH stop and SM-like Higgs masses lie in the ranges indicated by the analysis of ref. [14], so that the universe undergoes a sufficiently strong first order EWPT to prevent baryon number washout (we relax this assumption and consider a heavy sfermion sector, including both stops, everywhere else in this analysis). Consequently, we consider a scenario under which the leading CPV particle asymmetry generation occurs in the gaugino-Higgsino sector. In order to accommodate the lower bound on the lightest SM-like Higgs boson, the LH stop mass must be of order one TeV, thereby leading to Boltzmann suppression of LH stops and the production of large CPV asymmetries in the stop sector. In principle, one could counteract this suppression to some extent through resonant CPV stop processes wherein $m_{\tilde{t}_{L}} \sim m_{\tilde{t}_{R}}$, but our assumption of a heavy sfermion sector suppresses the importance of this contribution, even if resonant. For relatively large $\tan \beta$ leading to enhanced bottom and tau Yukawa couplings, one might introduce additional sources associated with these scalar quarks, but we leave consideration of this possibility to a future study. The remaining source of CPV asymmetries thus arises in the gaugino-Higgsino sector, for which one may consider relatively light superpartner masses (of order a few hundred $\mathrm{GeV}$ ). The corresponding CPV phases are, after appropriate field redfinitions:

$$
\phi_{j}=\operatorname{Arg}\left(\mu M_{j} b^{*}\right),
$$

where $\mu$ is the supersymmetric Higgsino-Higgs mass parmeter; $M_{j}$ are the SUSY-breaking soft mass parameters for the bino $(j=1)$ and wino $(j=2)$; and $b$ is a soft SUSY-breaking Higgs mass parameter. 
To obtain $Y_{B}$ as a function of the MSSM parameters, we rely on the work of ref. [12, 13, 15-17], which include the parameter dependence of both the CPV source terms in the quantum Boltzmann equations as well as that of the CP-conserving, particle numberchanging interactions. Both sets of effects are required for a robust prediction: the CPV source terms determine the overall scale of CPV Higgsino-Higgs asymmetries that may arise, while the particle number-changing interactions determine the efficiency with which these asymmetries convert into the left-handed fermion density that biases the electroweak sphalerons. To lowest order in Yukawa and gauge couplings, particle number changing processes occur through decays and inverse decays $A \leftrightarrow B+C$; in regions where these reactions are kinematically forbidden, $A+B \leftrightarrow C+D$ scattering reactions dominate. We avoid these parameter space regions as there does not yet exist a complete study of the parameter dependence of these scattering rates.

For the CPV sources, we also rely on the computation of ref. [15], which computed the sources to leading non-trivial order in the spacetime varying Higgs background field. This approximation is likely to overestimate the source strength, ${ }^{1}$ so we consider our results for $Y_{B}$ to represent the most optimistic scenario, and therefore, the most conservative for statements about the impact of EDMs. On-going theoretical work is aimed at resumming the background field while consistently including the effects of diffusion ahead of the bubble wall and interactions that would drive the out-of-equilibrium plasma toward equilibrium. Once such a consistent framework has been achieved, our analysis will likely need to be up-dated.

We also note that the sources of ref. [15] apply to only resonant production of CPV Higgsino asymmetries, and we do not consider non-resonant processes that generally require substantially larger CPV phases in order to produce the observed $Y_{B}$ (see, e.g. ref. [18]). Resonant EWB occurs when the mass parameters of two particles having a trilinear coupling with the Higgs scalar are nearly degenerate. In the case of top squarks, for example, resonant asymmetry production occurs when for nearly equal, and light enough LH and RH stop masses, a situation precluded by the requirements of a strong first order EWPT and the bound on the lightest Higgs mass as noted above. For the gauginos and Higgsinos, resonant EWB arises when $\mu \sim M_{1,2}$ - the scenario on which we concentrate here. ${ }^{2}$

Within this context, the overall source strength depends crucially on both the CPV phases and $\Delta \beta$, the change in $\tan ^{-1} v_{u} / v_{d}$ from the bubble wall exterior to its interior with $v_{u}$ and $v_{d}$ being the spacetime-dependent vacuum expectation values (or background fields) associated with the neutral up- and down-type Higgs scalars, respectively. The authors of ref. [21] showed that $\Delta \beta$ decreases with increasing $m_{A}$, and we take this $m_{A}$-dependence into account in our analysis of the parameter space. Doing so is not entirely consistent with the phase transition analysis of ref. [14], which considered $m_{A}$ larger than $\sim 10 \mathrm{TeV}$. We expect that a two-loop EWPT analysis allowing for a dynamically active, light CP-odd Higgs would not change the allowed ranges of $m_{\tilde{t}_{R}}$ and $m_{h}$ substantially, and we therefore consider values of $m_{A}$ below one $\mathrm{TeV}$ as needed for unsuppressed, resonant, CPV sources.

The dependence of $Y_{B}$ on $\tan \beta$ remains a topic of on-going research. To our knowledge,

\footnotetext{
${ }^{1}$ See, e.g. refs. [18-20].

${ }^{2}$ In our conventions, the Higgs and Higgsino fields have been rotated so that $\mu$ is real and positive.
} 
there exist no results in the literature, for the $\tan \beta$-dependence of $\Delta \beta$. On the other hand, a previously overlooked $\tan \beta$-dependence of transport dynamics has been observed in refs. $[12,13]$. Earlier work had assumed that the CP-conserving, particle number changing processes were dominated by stop quark (squark) Yukawa interactions, given the large value of the top Yukawa coupling relative to that of the other (s)fermions. For moderate $\tan \beta$, however, the (s)bottom and (s)tau Yukawa couplings are relatively enhanced, and their impact on the conversion of a Higgs-Higgsino asymmetry into the net left-handed fermion density $\left(n_{\text {left }}\right)$ that couples to electroweak sphalerons can be substantial. In what follows, we include this $\tan \beta$ - dependence by re-scaling $Y_{B}$ according to figure 1 of ref. [17], that shows the ratio of $Y_{B}$ computed including the bottom and tau Yukawa couplings to the baryon asymmetry computed in their absence as a function of $\tan \beta$. For purposes of this analysis, we work in the limit of heavy $\tilde{\tau}_{R}$, but note that - as shown in figure 1 of ref. [17] — the presence of a light $\tilde{\tau}_{R}$ can lead to a suppression of $Y_{B}$. We also work in the region of parameter space wherein $m_{\tilde{b}_{R}} \gg m_{\tilde{t}_{R}}$ to avoid further suppression of $Y_{B}$ or even a sign change that can occur for $m_{\tilde{b}_{R}} \lesssim m_{\tilde{t}_{R}}$.

For the computation of bounds from EDMs, we draw on the two-loop computations of ref. [10]. Limits from one-loop EDMs generally imply values of $\left|\sin \phi_{j}\right|$ that are too small to accommodate the observed value of $Y_{B}$, even in the regions of resonant EWB. To circumvent these bounds, we work in the limit of heavy first generation sfermions, thereby suppressing one-loop EDMs without quenching $Y_{B}$ via Boltzmann suppression of stops, gauginos, and Higgsinos. In this limit, both the elementary fermion EDMs and the chromo-EDMs of quarks are suppressed. As a result, constraints from the ${ }^{199} \mathrm{Hg}$ atomic EDM limit are minimal, as the ${ }^{199} \mathrm{Hg}$ atomic EDM is dominated by the chromo-EDMs in the MSSM. In contrast, significant bounds from the two-loop EDMs of the electron and quarks (contributing to $d_{n}$ ) can occur, with the most stringent arising from graphs involving closed chargino and neutralino loops and the exchange of two bosons as in figure 1 of ref. [10]. To analyze these constraints, we rely on the work of ref. [10] and consider two cases:

(a) universal gaugino phases, wherein $\phi_{1}=\phi_{2}$, and

(b) non-universal phases, for which $\phi_{1}$ and $\phi_{2}$ are allowed to differ.

For the second scenario, the sensitivity of the electron and neutron EDMs to $\phi_{2}$ is roughly fifty times stronger than the sensitivity to $\phi_{1}$.

In summary, the most optimistic scenario leaving the largest window for viable MSSM EWB occurs in the following region of parameter space: a light right-handed stop with $m_{\tilde{t}_{R}} \lesssim 125 \mathrm{GeV}$; the remaining sfermion masses being heavy, $m_{\tilde{f}} \gtrsim 1 \mathrm{TeV}$; light gauginos and Higgsinos with $\mu \sim M_{1}$; non-universal phases: $\phi_{1} \neq \phi_{2}$; a relatively light pseudoscalar Higgs and moderate to small $\tan \beta$. In what follows, we illustrate the impact of relaxing these assumptions.

The MSSM parameter space compatible with EWB is severely constrained by the requirement that the lightest neutralino be the lightest supersymmetric particle (LSP), since a light RH stop with $m_{\tilde{t}_{R}} \lesssim 125 \mathrm{GeV}$ forces the LSP mass to also be below that 
value. ${ }^{3}$ However, the requirement of a light $\mathrm{RH}$ stop can be relaxed in several minimal extensions to the MSSM that include for instance an extended Higgs sector. One large class of such models are those that include a gauge singlet with couplings to the $\mathrm{SU}(2)$ Higgs doublets $[22,23]$. Other viable beyond-the-MSSM setups featuring a strongly first order EWPT also include models with a fourth fermion generation [24] and top-flavor models [25]; models with higher dimensional scalar representations of SU(2) (which might lead to a multistep EWPT [26]) provide yet another promising such framework [27, 28]. In several of these models, the physical mechanism that drives a strongly first order EWPT is decoupled from EWB as the source of the BAU. As such, in what follows we will assume that the $\mathrm{RH}$ stop be at the $\mathrm{TeV}$ scale, and that a physical process that does not interfere with EWB drives a strongly first order EWPT. This assumption will allow us to explore wider regions of the parameter space relevant to EWB, although we shall also show the regions that would correspond to an LSP lighter than $125 \mathrm{GeV}$ (dashed blue lines in figure 1 and 5). From now on, in this manuscript we take all sfermions, including the RH stop, to be heavy, $m_{\tilde{f}} \gtrsim 1 \mathrm{TeV}$.

\section{Baryogenesis and neutralino relic (over-)abundance}

If the entropy density of the Universe is not a constant after the freezeout of the net baryon density, the resulting baryon asymmetry today will in general be affected. While episodes such as very low-scale inflation (as low as the electroweak scale) have been envisioned [2931], this is not the standard paradigm. However, an entropy injection episode might actually be needed in a successful theory of supersymmetric electroweak baryogenesis. We provide here an example and study the implications for the underlying supersymmetric theory.

In the framework we consider here, where $R$-parity is conserved, the LSP is stable. Given null results in searches for a stable massive charged or strongly-interacting particle, ${ }^{4}$ the LSP must be neutral, and its density today must be compatible with (namely, at least not exceed) the universal matter density to avoid over-closing the Universe. As such, a relic, neutral LSP $\chi$ must have abundance $\Omega_{\chi} h^{2}$ (in units of the critical density) less or equal to the dark matter density $\Omega_{\mathrm{DM}}$. If this is not the case, either the underlying supersymmetric theory is ruled out or a mechanism must exist that dilutes away the excessive $\chi$ relic abundance.

Weakly interacting massive particles, such as the lightest neutralino in MSSM scenarios where it is the LSP, undergo a rather universal (i.e. independent of the details of masses and interactions) freezeout process from the thermal bath in the early universe that occurs around temperatures $T_{\text {freeze-out }} \sim m_{\chi} /(20 \div 25)$. In models of successful electroweak baryogenesis, where the LSP cannot be heavier than $\sim 1 \mathrm{TeV}$ [33], the freezeout temperature is thus always below $50 \mathrm{GeV}$. Therefore, freeze-out occurs typically at much lower temperatures than those relevant for the electroweak phase transition. In turn, this implies

\footnotetext{
${ }^{3} \mathrm{~A}$ caveat is the assumption of a gravitino LSP, but we do not consider this possibility here.

${ }^{4}$ In particular, the number density of a metastable negatively charged electroweak-scale particle $\chi^{-}$relative to entropy is constrained to be smaller than $n_{\chi^{-}} / s \leq 3 \times 10^{-17}$, since it leads to catalytic enhancement of the ${ }^{6} \mathrm{Li}$ production [32].
} 
that if an entropy injection episode is responsible for the dilution of an excessive thermal relic density of neutralinos, the same entropy injection episode will also dilute away the baryon number density produced at the electroweak phase transition.

We are therefore left with two possibilities: either models with an excessive neutralino thermal relic abundance are ruled out, or they are salvaged by a modified cosmological expansion that inflates them away. ${ }^{5}$ However, if this second possibility is realized, the net baryon asymmetry will also be diluted away, requiring a larger asymmetry to be produced at the electroweak phase transition. It is this latter possibility that we explore in this section.

To illustrate the effect of enforcing a cosmologically acceptable density of relic neutralinos, we consider in the upper two panels of figure 1 the thermal relic density of neutralinos in the $\left(M_{1}, \mu\right)$ plane, for a scenario with heavy sfermions, $\tan \beta=10, M_{2}=2 \times M_{1}=M_{3} / 3$ and $m_{A}=150 \mathrm{GeV}$ (top left panel) and $500 \mathrm{GeV}$ (top right panel). The green line corresponds to a thermal relic abundance in accord with the inferred density of cosmological dark matter. In the lower right corners of these two plots, neutralinos only constitute a fraction of the cosmological dark matter (unless some non-thermal production mechanism is operative). Although this would mean one needs to postulate an additional particle to provide the rest of the dark matter density, an under-abundance of thermal neutralinos is cosmologically viable, and there is no need to rescale the baryon asymmetry that gets produced in those regions of the parameter space. The blue dashed lines in the upper panels indicate a lightest neutralino mass of $125 \mathrm{GeV}$.

In the lower right corner $\mu \lesssim M_{1}$, and the lightest neutralino is higgsino-like, and it efficiently pair-annihilates into $W^{+} W^{-}$ad $Z Z$ pairs. Further contribution to the efficient pair-annihilation processes in the early Universe also stems from the co-annihilation of the quasi-degenerate next-to-lightest higgsino-like neutralino and chargino. In the upper right portion of the plots, the increasing bino-like content of the lightest neutralino mass eigenstate suppresses annihilation to the $\mathrm{SU}(2)$ gauge bosons, and only if a resonant annihilation channel occurs (e.g. for $m_{\chi} \sim m_{A} / 2$ in the left plot) can the relic abundance be compatible with the upper limit to the cosmological dark matter density. In that region, therefore, we will need to rescale the neutralino density by at least a factor $\Omega_{\chi} / \Omega_{\mathrm{DM}}$, the former factor being the thermal neutralino relic density in units of the critical density.

Without accounting for such rescaling, the middle two panels in figure 1 show the values of $\sin \phi$ one would need to produce the observed baryon asymmetry of the Universe. Each contour of the double funnel-like region corresponds to a given value of $\sin \phi$, where we take $\phi_{1}=\phi_{2} \equiv \phi$ under the assumption of phase universality. Going from the outermost funnel to the innermost, successive contours correspond to the values of $\sin \phi$ indicated in the panels "isolevels"). The behavior of the contours is consistent with the results given in figure 1 of our earlier work [33]. Increasing the value of $m_{A}$ suppresses the produced net baryon asymmetry, and therefore the viable regions (those inside the funnels corresponding to the resonant regions where $\mu \sim M_{1}$ or $\sim M_{2}$ ) shrink.

Accounting for the neutralino number density dilution and transferring the dilution to

\footnotetext{
${ }^{5}$ Interestingly, such a mechanism can be built in the electroweak phase transition itself, see e.g. the recent analysis of ref. [34].
} 

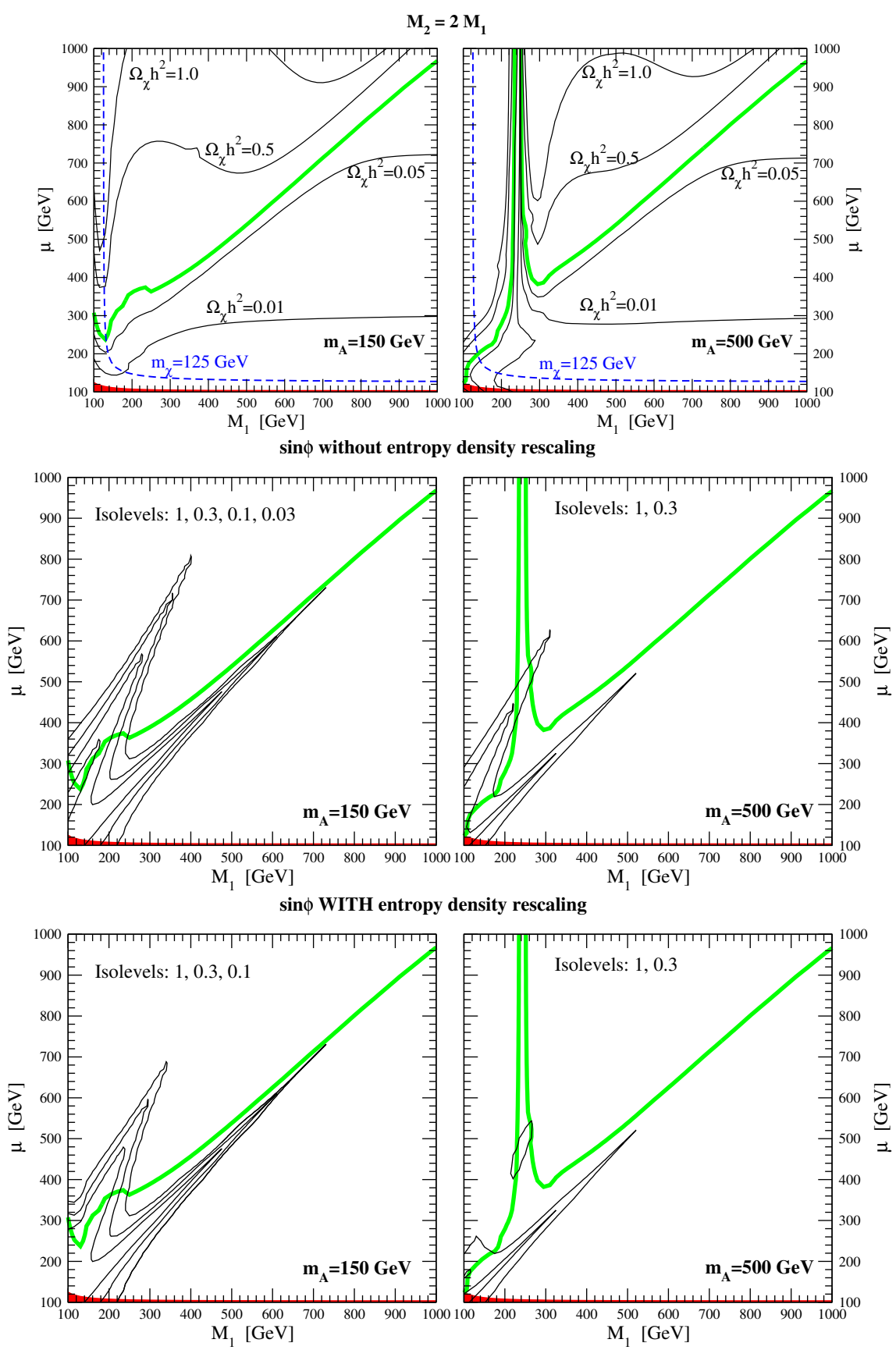

Figure 1. Iso-level curves for the lightest neutralino relic abundance (upper panels) and for the gaugino-higgsino CPV phase producing the central value of the BAU, without (central panels) and with (lower panels) entropy rescaling for models with over-abundant relic neutralinos. All panels refer to the $\left(M_{1}, \mu\right)$ plane, with $m_{A}=150 \mathrm{GeV}$ in the left panels, and $m_{A}=500 \mathrm{GeV}$ for those to the right. The red shaded region is excluded by the non-observation of light neutralino pairs at LEP. The green bands correspond to a neutralino relic density consistent with the WMAP results. The blue dashed lines in the upper panels indicate a lightest neutralino mass of $125 \mathrm{GeV}$. 

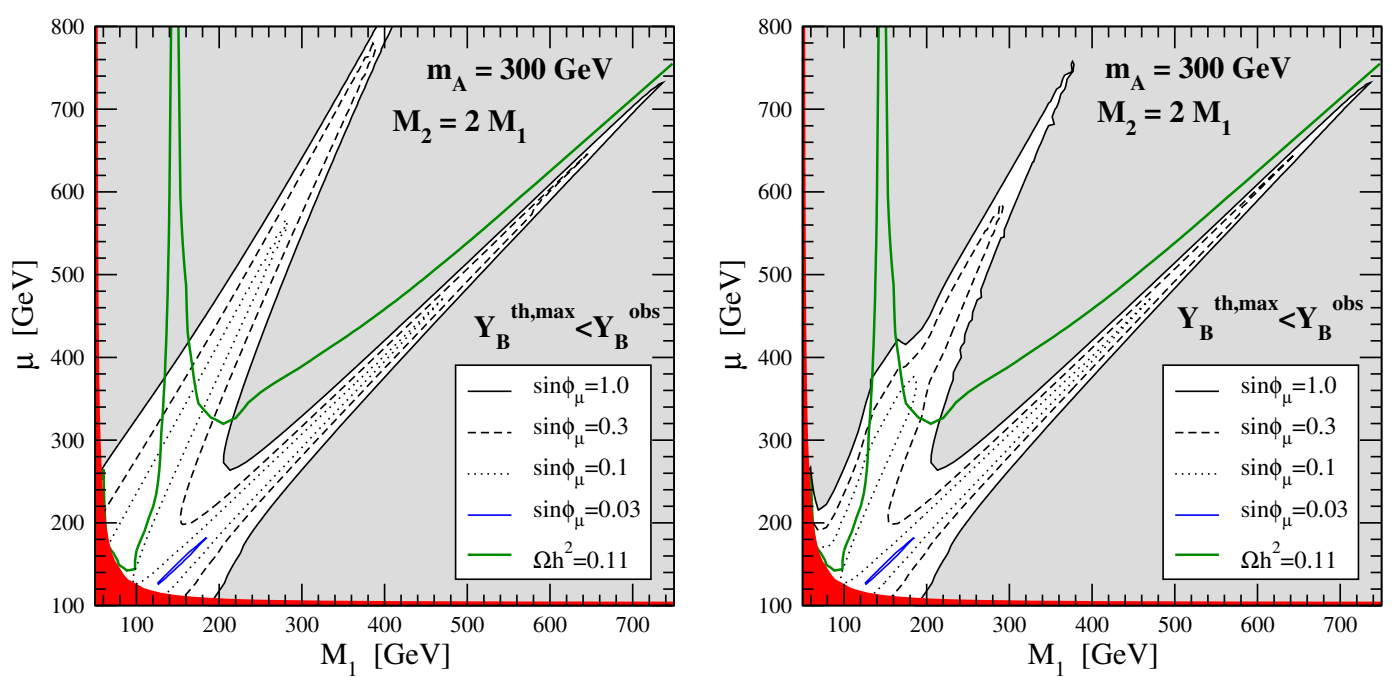

Figure 2. Curves at constant CPV gaugino-higgsino phase, on the $\left(M_{1}, \mu\right)$ plane, at $m_{A}=300 \mathrm{GeV}$ and $M_{2}=2 M_{1}$, with (right) and without (left) entropy rescaling for overabundance relic neutralinos models. The grey region does not produce a large enough BAU through resonant processes, the red region is excluded by LEP searches for the lightest chargino, and the green lines indicate a neutralino relic abundance equal to the cold dark matter abundance in a standard cosmological setup.

the baryon number asymmetry as well leads to a requirement of larger $\sin \phi$ (i.e. a larger initial net baryon number density) in regions where $\Omega_{\chi} \gtrsim \Omega_{\mathrm{DM}}$. This feature is illustrated in the bottom two panels of figure 1. There, we find a suppression of the $M_{2} \sim \mu$ funnel, where the lightest neutralino is dominantly bino-like. For large values of $m_{A}$ (lower left panel), said funnel essentially disappears, leaving bino-driven electroweak baryogenesis [11] as the only available option.

In the following section we further explore the phenomenological consequences of entertaining a viable supersymmetric model from the standpoint of cosmology, and we derive lower limits on the electron and neutron EDM in the present scenario.

\section{A lower limit on the electron and neutron EDM}

Figure 2, left, reiterates what is shown in the figure 1, but this time for a heavy Higgs sector set to $m_{A}=300 \mathrm{GeV}$. As in the previous plots, we again assume here $M_{2}=2 M_{1}$. The different shape in the contours depend on the assumed values for $m_{A}$. Again, the green lines indicate a thermal neutralino relic density in accord with the inferred cosmological dark matter density. The funnel where the lightest neutralino resonantly annihilates through the s-channel on-shell exchange of one of the heavy neutral Higgses appears near $M_{1} \sim m_{A} / 2=150 \mathrm{GeV}$. The left panel shows contours of constant values for the gauginohiggsino phase needed to have the right amount of baryon asymmetry, neglecting the overabundance of neutralinos in the upper-left part of the parameter space. As indicated by the right panel, the effect of the required entropy dilution shrinks the available regions for those values of $\left(M_{1}, \mu\right)$ where $\Omega_{\chi} \gtrsim \Omega_{\mathrm{DM}}$. 

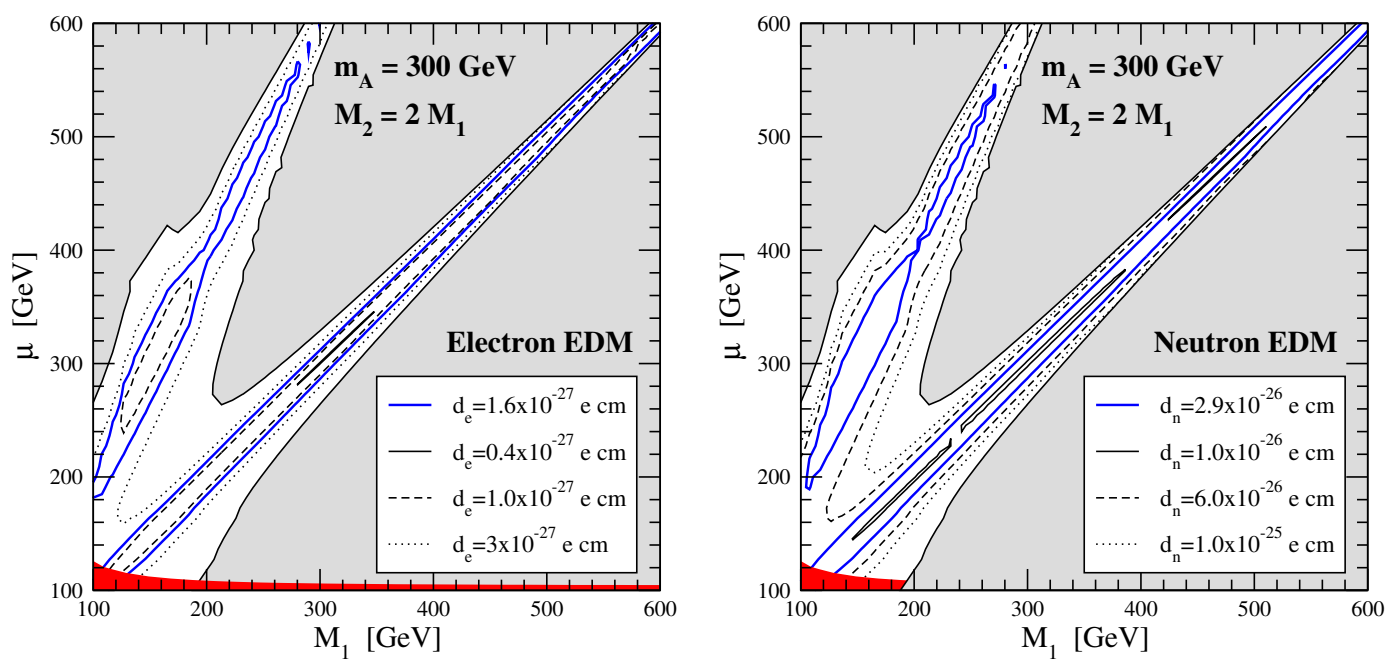

Figure 3. Curves of constant values for the electron (left) and for the neutron (right) electric dipole moment, on the same plane as figure 2, right. The regions outside the blue contours are excluded by current experimental limits.

Focusing now on the physically motivated case of a re-scaled net baryon asymmetry, figure 3 shows lines of constant values for the electron (left) and for the neutron (right) EDMs. As one would expect, the largest values occur where the $\mathrm{CP}$ violating phases are largest, i.e. near the outmost boundaries of the funnel regions, while smaller values are obtained in the central part of the funnels: there, the resonant source term allows for a lower $\mathrm{CP}$ violating phase, resulting in smaller electric dipole moments. The only viable regions are therefore those inside the blue contours. In summary, we find for the slice of parameter space we consider that there is a lower limit to the electric dipole moment of the electron around $0.4 \times 10^{-27} \mathrm{e} \mathrm{cm}$, roughly a factor 4 below the current experimental limit; the analogous limit for the neutron $\mathrm{EDM}$ sits at $10^{-26} \mathrm{e} \mathrm{cm}$, i.e. only a factor 3 below current sensitivity. We emphasize that these lower limits pertain only to scenarios involving universality of phases: $\operatorname{Arg}\left(\mu M_{1} b^{*}\right)=\operatorname{Arg}\left(\mu M_{2} b^{*}\right) \equiv \phi_{\mu}$. In section 5, we will relax this unnecessary assumption.

Up to this point, we have considered only the (complete set [10]) two-loop contributions to the EDMs, since one-loop contributions are negligible for sufficiently heavy 1st generation sfermion masses. The following figure 4 addresses the question of how small the sfermion mass scale must be for one loop contributions to become dominant over the two-loop ones. The left and right panels of figure 4 focus on the case of the EDM of the electron and of the neutron, respectively. We set all CP violating phases to zero with the sole exception of the relative higgsino-gaugino phase, as in the rest of this study. The sfermion scale we refer to here alludes naturally to the relevant sfermions, i.e. first generation sleptons and squarks. Roughly, the size of the CP violating phase factors out from both the one- and two-loop contributions. The larger the values of $\left(M_{1}, \mu\right)$, the more suppressed the two-loop contributions: as a result, the sfermion mass scale that would produce a comparable EDM can also 

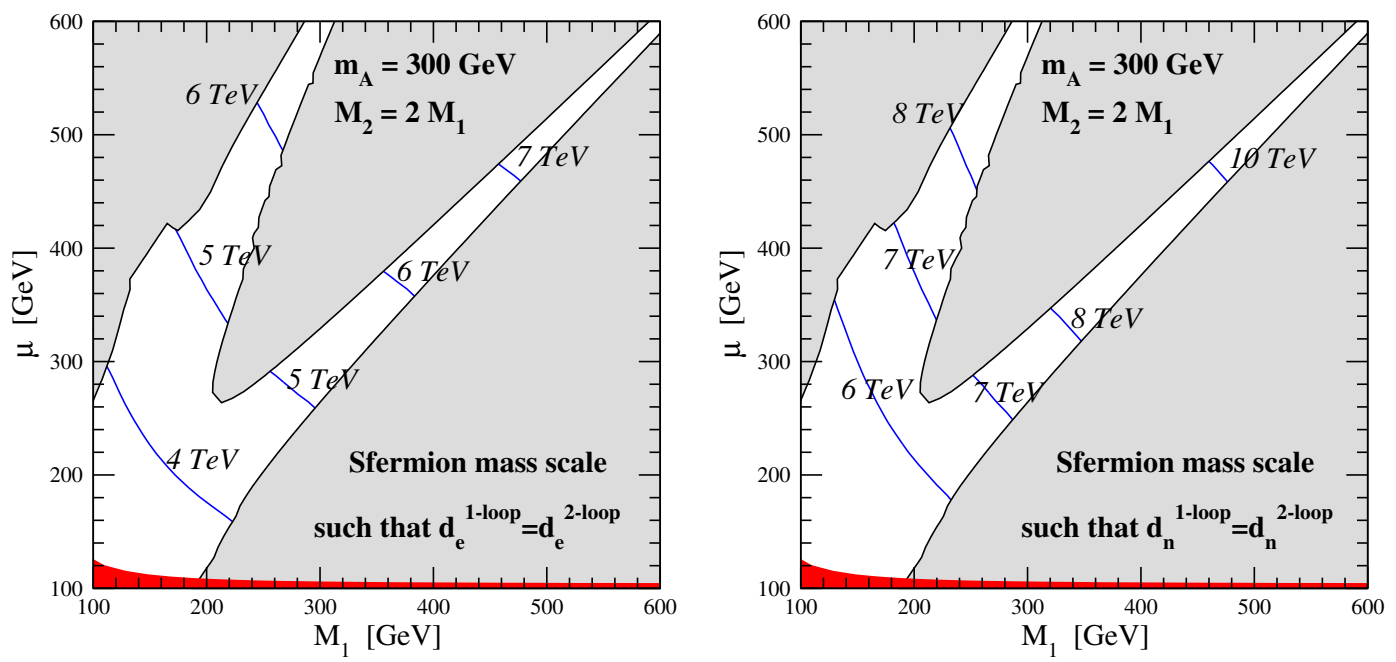

Figure 4. Curves indicating the values of a common sfermion mass scale such that one and two loop contributions to the electron (left) and to the neutron (right) EDM are equal, on the same plane as figure 2, right. At a given sfermion mass scale indicated on the lines, points inside the funnel region lying closer to the origin correspond to two-loop contributions that are larger in magnitude than one-loop contributions. The converse holds for points lying closer to the funnel tips.

be larger, as the iso-level contour labels show. Fixing the sfermion mass scale to e.g. $6 \mathrm{TeV}$, points to the upper-right of the lines have a larger one-loop contribution than the two-loop one, while points to the lower-left side of the lines have a larger two-loop contribution.

In summary, we find that the one-loop contributions to the electron EDM start to be significant when the mass scale is around $4-7 \mathrm{TeV}$, while squarks as heavy as $10 \mathrm{TeV}$ can give significant contributions at the one-loop level to the neutron EDM. This implies, in particular, that the neutron EDM is overall more sensitive to one-loop contributions than the electron EDM

\section{$5 \quad \mathrm{EDM}$ in the bino-driven electroweak baryogenesis scenario}

As pointed out by some of us in ref. [11], it is possible for MSSM baryogenesis to remain viable even if the next generation of EDM searches yield null results. Theoretically, this scenario requires that one relaxes the assumption of universality between the relative binohiggsino and wino-higgsino phase, i.e. $\phi_{1} \neq \phi_{2}$ and work in the limit of heavy 1st generation sfermion masses. In this way, as long as $\phi_{2}$ is sufficiently small, EDMs are suppressed, while bino-driven electroweak baryogenesis, efficient if the resonant condition $\mu \sim M_{1}$ is approximately valid, can proceed with large bino-higgsino phases $\phi_{1}$. The latter, as shown and explained in detail in ref. [11], does not lead to excessively large contributions to the EDMs, even if $\left|\sin \phi_{1}\right|$ is of order one.

In the bino-driven portion of the parameter space, $\Omega_{\chi} \lesssim \Omega_{\mathrm{DM}}$, and no entropy dilution is needed to wash out excess relic neutralinos. We illustrate this point in the left panel of figure 5, where we assume $\phi_{2}=0$ and show the values of $\phi_{1}$ for which we generate the 

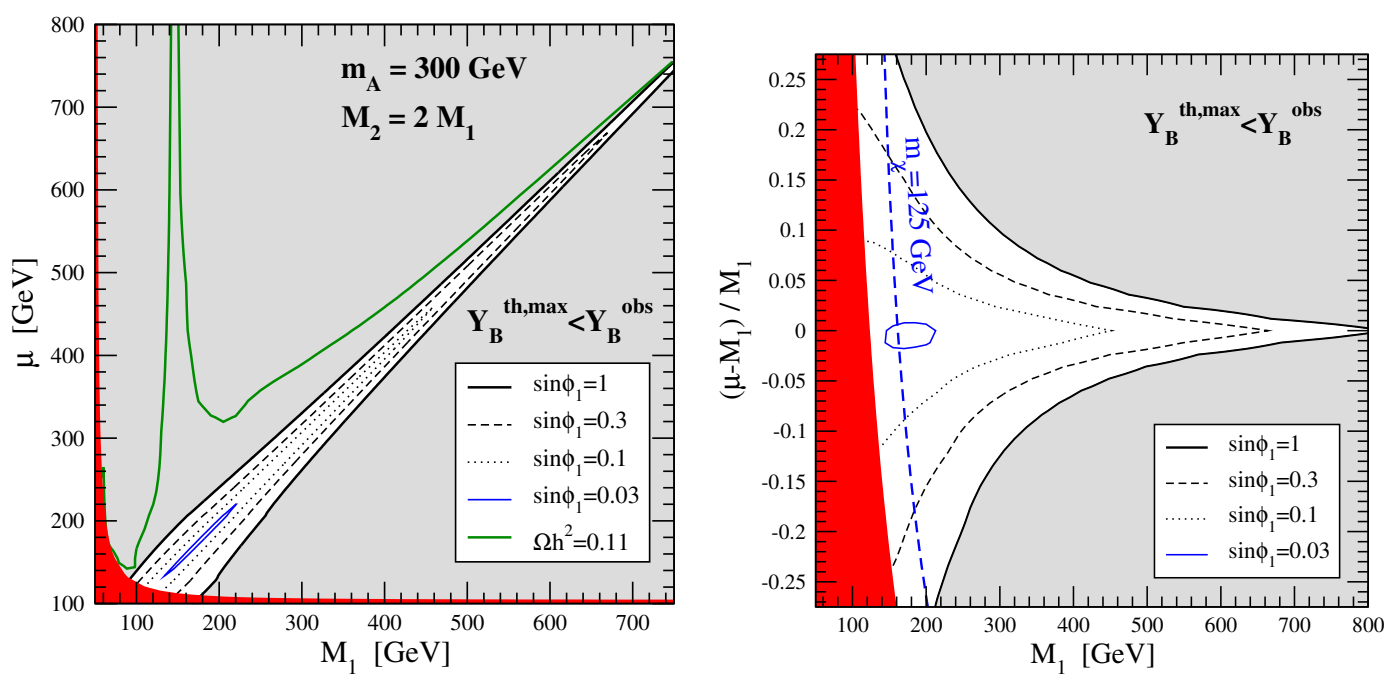

Figure 5. Curves indicating constant values of the relative bino-higgsino phase $\phi_{1}$ that produces the right BAU through resonant processes, on the same plane as figure 2 (left), and on the plane defined by $M_{1}$ and by the relative bino-higgsino mass splitting $\left(\mu-M_{1}\right) / M_{1}$. The grey region does not produce a large enough BAU through resonant processes, the red region is excluded by LEP searches for the lightest chargino. In the left panel, the green lines indicate a neutralino relic abundance equal to the cold dark matter abundance in a standard cosmological setup. In the right panel, the blue dashed line indicates a lightest neutralino mass of $125 \mathrm{GeV}$.

observed baryon asymmetry. Since resonant bino-driven EWB is most efficient in the region for $\mu \sim M_{1}$, it is useful to re-cast our results in terms of $M_{1}$ and the relative mass-splitting $\left(\mu-M_{1}\right) / M_{1}$. In the right panel of figure 5 , we show the bino-driven region (i.e. the region where $\left.\mu \sim M_{1}\right)$ in the $\left(M_{1},\left(\mu-M_{1}\right) / M_{1}\right)$ plane. As the contours for successively smaller values of $\sin \phi$ indicate, a lighter overall mass scale and smaller relative mass splitting are needed to reproduce the observed $Y_{B}$ as $\sin \phi$ is decreased.

We employ the same parameter space (the $\left(M_{1},\left(\mu-M_{1}\right) / M_{1}\right)$ plane) to illustrate that even in the case of bino-driven EWB we obtain an absolute lower limit on the size of the electron and the neutron EDM that would be consistent with this scenario. Here we give contours of constant $d_{e}$ (left panel) and $d_{n}$ (right panel), rather than constant $\sin \phi_{1}$, consistent with $Y_{B}$. The contours in the left panel of figure 6 , left, indicate that the minimal electron EDM is around $10^{-29}$ e cm, roughly a factor 200 below present limits. The projected sensitivity improvement to cover this slice of the bino-driven electroweak baryogenesis scenario with neutron EDM searches is instead of around a factor 100, as shown in the right panel.

\section{The $\left(\tan \beta, m_{A}\right)$ plane}

Thus far, our exploration of the supersymmetric parameter space compatible with EWB has focused largely on the parameter space defined by the masses of those particles directly entering the source terms in the production of the baryon asymmetry at the electroweak 

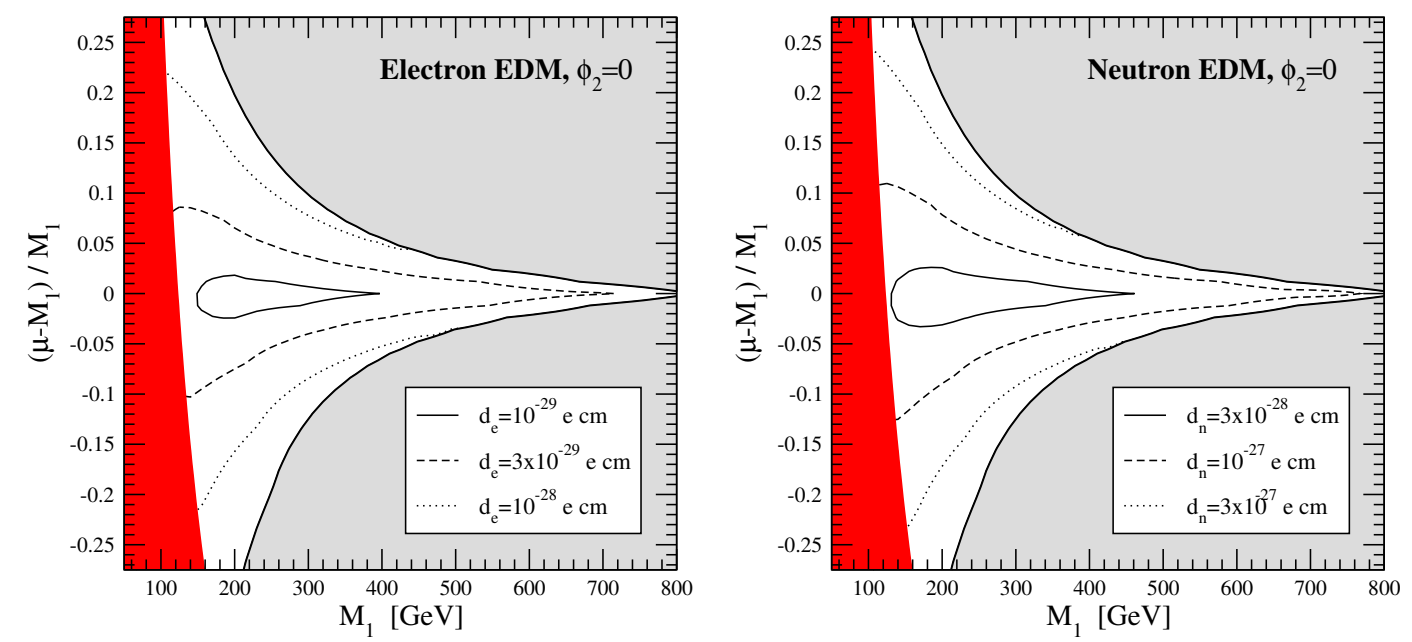

Figure 6. Curves indicating constant values for the electron (left) and for the neutron (right) electric dipole moment, on the same plane as figure 5, right. The CPV phase $\phi_{1}$ is set to the value giving the right $\mathrm{BAU}$, while $\phi_{2}$ (the relative wino-higgsino phase) is set to 0 .

phase transition. We now turn our attention to two other parameters that play a crucial role in the dynamics of baryon number generation at electroweak temperatures: the ratio of the vacuum expectation values of the neutral componets of the two SU(2) Higgs doublets, $\tan \beta$, and the mass of the CP-odd Higgs, $m_{A}$. The former enters in the calculation of $Y_{B}$ via not only directly in the sources via the various particle couplings, including Yukawa couplings, but also via the effects described in ref. $[12,13,17]$. In the latter work, it was observed that for moderate values of $\tan \beta$, the timescale for $\mathrm{b}$ (squark) and tau (s)lepton Yukawa interactions can be shorter than the timescale associated with diffusion ahead at the bubble wall. Consequently, for sufficiently light right-handed sbottoms and staus, the dynamics of these sfermions can substantially alter the transport dynamics that govern the conversion of Higgs-Higgsino CP-violating asymmetries into the (s)quark sector asymmetries. The mass parameter $m_{A}$ also enters in the calculation of $Y_{B}$ through the parameter $\Delta \beta$, to which $Y_{B}$ is linearly proportional. Here, we treat the dependence of $Y_{B}$ on $m_{A}$ according to the two-loop results presented in [21], and the dependence on $\tan \beta$ according to the full treatment outlined in $[12,13] .{ }^{6}$

So far, the $\left(\tan \beta, m_{A}\right)$ parameter space for this problem has not been extensively explored. For illustrative purposes, we adopt here the universal phase assumption and we show that there can be strong bounds in this space that have not been emphasized in previous work. At the end of this analysis, we discuss briefly our expectations for the case of non-universal phases.

In figure 7 , we show the constraints on the $\left(\tan \beta, m_{A}\right)$ parameter space from various phenomenological and cosmological considerations. We set the gaugino and higgsino mass parameters to $M_{1}=145 \mathrm{GeV}, M_{2}=290 \mathrm{GeV}$ and $\mu=300 \mathrm{GeV}$. As discussed above, we

\footnotetext{
${ }^{6}$ Note that we do not consider the effects of varying $m_{A}$ on the strength of the electroweak phase transition since in the latest work of ref. [9], the pseudoscalar A was integrated out of the effective theory.
} 


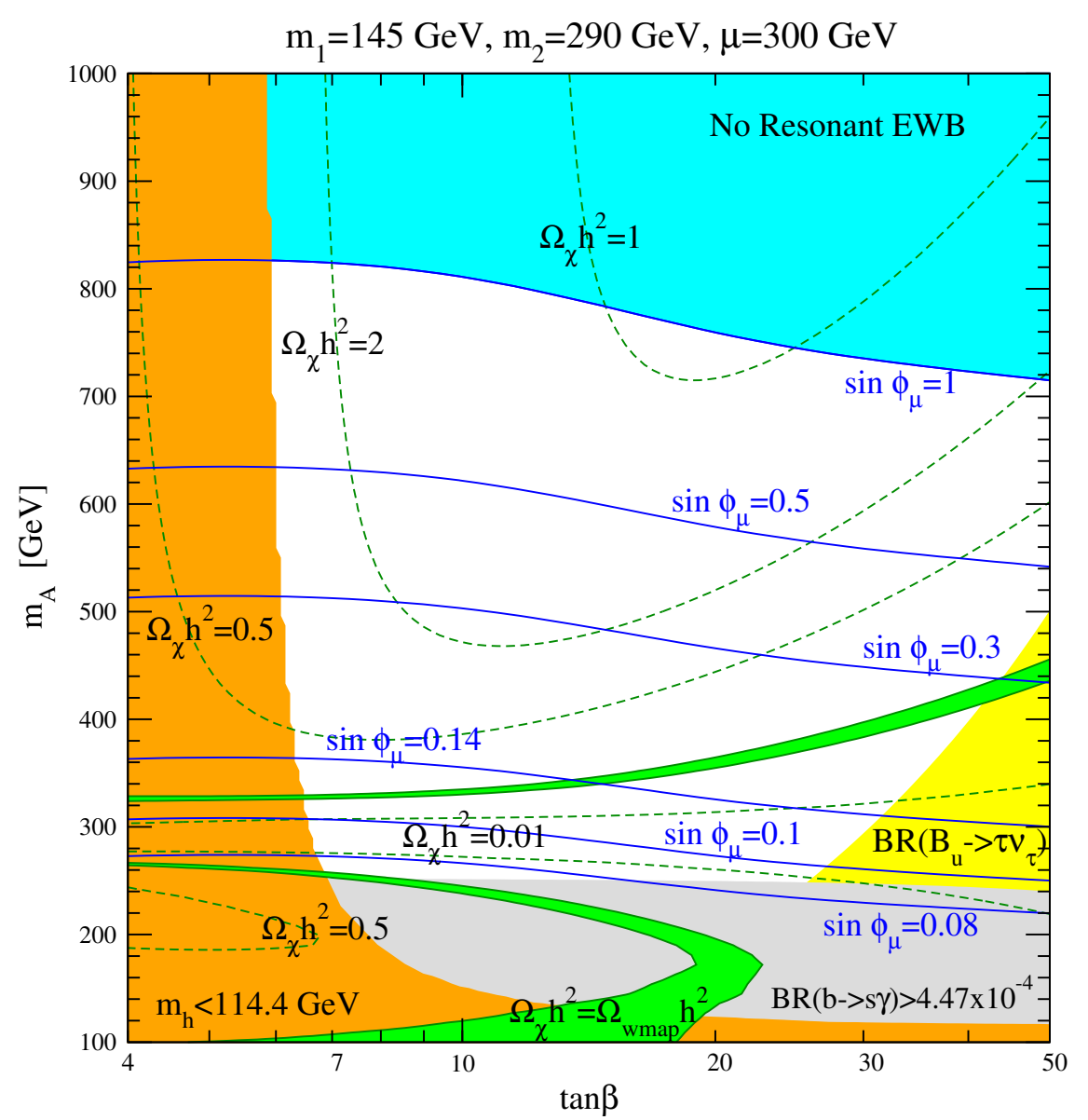

Figure 7. The $\left(\tan \beta, m_{A}\right)$ plane: the orange region is ruled out by LEP limits on the lightest Higgs, while the grey region is ruled out by excessive supersymmetric contributions to the inclusive $b \rightarrow s \gamma$ branching ratio and the yellow region is ruled out by bounds on the $\operatorname{BR}\left(B_{u} \rightarrow \tau \nu_{\tau}\right)$. In the blue region at the top the generated BAU is too small, while the green contours indicate the 2- $\sigma$ preferred range for relic neutralinos to be the dark matter. We also show contours of constant values for the neutralino relic abundance (green dashed lines) and for the value of the gaugino-higgsino CPV phase $\sin \phi_{\mu}$ (solid blue lines) such that electroweak baryogenesis successfully accounts for the BAU.

also assume a common gaugino-higgsino phase $\phi=\operatorname{Arg}\left(\mu M_{j} b^{*}\right), j=1,2$. In the orange region to the left and bottom of the plane, the Higgs mass is lower than the limit set by LEP-II of $114.4 \mathrm{GeV}$ (this limit applies for the heavy sfermion sector we consider in the present analysis). In addition, in the grey area the supersymmetric contribution to the $b \rightarrow s \gamma$ decay exceeds the experimentally allowed value.

At large values of $\tan \beta$, a significant constraint stems from MSSM contributions to the $B_{u} \rightarrow \tau \nu_{\tau}$ decay mode $[35,36]$. This branching ratio, in turn, depends on SUSYQCD corrections to the charged Higgs boson coupling to fermions. These corrections are asymptotically small in the limit of heavy sfermions, and, for our choices of parameters, the relevant quantity $\epsilon_{0}$ in eq. (6) of ref. [35] is always smaller than 0.01. Given that in the present analysis we mostly focus on the limit of heavy sfermions to avoid one loop 
contributions to the EDMs, we set $\epsilon_{0}=0$, which entails slightly more stringent constraints than for a small, finite positive value. Imposing the $2-\sigma$ limit to the ratio of the MSSM to SM contribution $0.53<R_{\tau \nu_{\tau}}<2.03$ [35], two disconnected regions of the $\left(\tan \beta, m_{A}\right)$ parameter space are ruled out. As shown in ref. [36], the portion of parameter space between these disconnected regions is actually also ruled out by constraints on MSSM contributions to the ratio of $K \rightarrow \mu \nu_{\mu}$ and $\pi \rightarrow \mu \nu_{\mu}$ branching ratios. For the range of $\tan \beta$ shown in figures $7-10$, the latter region also lies within the $b \rightarrow s \gamma$ exclusion region. We shade in yellow the remaining excluded parameter space implied by the $B_{u} \rightarrow \tau \nu_{\tau}$ constraints.

The green dashed lines correspond to various, constant values of the lightest neutralino relic abundance $\Omega_{\chi} h^{2}$ (as indicated by the labels). Within the thick green bands, the thermal neutralino relic abundance is within the $2 \sigma$ WMAP range for the average cold dark matter density. Finally, the blue lines indicate which value of the phase $\phi$ is needed for the particular point in parameter space to produce the observed $Y_{B}$. For these lines, we do not include the dilution of over-abundance neutralinos. In the upper region of the plot, shaded in cyan, no resonant electroweak baryogenesis can produce enough baryon asymmetry, even neglecting the effect of entropy dilution: this therefore sets, for the particular values of gaugino and higgsino parameters we picked here, an upper limit of $m_{A} \leq 800 \mathrm{GeV}$.

In figure 7 , as in the remainder of this paper unless otherwise specified, we have assumed heavy squarks and we have neglected the effect of down-type sfermions (effectively assuming heavy sbottoms and staus). We study in figure 8 the impact of two effects:

(i) rescaling of the net baryon asymmetry for parameter space points with a relic thermal neutralino abundance in excess of the dark matter density, and

(ii) factoring in finite masses for third generation sfermions.

The lines in figure 8 represent iso-level contours of $\phi$ (corresponding to the observed $Y_{B}$ ) under different assumptions. The red lines correspond to the limit of super-heavy downtype sfermions, after rescaling for over-dense neutralino relic densities. As evident, the parameter space shrinks with respect to the case without density rescalling (figure 7), and for reasonably low values of $\tan \beta$, the maximal value $m_{A}$ can take is below $600 \mathrm{GeV}$. Note that in contrast to the isolevel contours of figure 7 , those of figure 8 increase monotonically with $\tan \beta$.

The black lines give the isolevel curves when the effect of bottom and top Yukawa interactions are included in the transport dynamics, for right-handed sbottom and stau masses equal to $1 \mathrm{TeV}$. Inclusion of these $\mathrm{RH}$ sfermions suppresses the baryon asymmetry, implying a need for a lighter $m_{A}$ for a given value of $\sin \phi_{\mu}$. The impact of the RH third generation sfermions is even more pronounced when they are relatively light. To illustrate, we show in green the isolevel contours for $300 \mathrm{GeV} \tilde{b}_{R}$ and $\tilde{\tau}_{R}$ masses. The appearance of "elbows" in these contours arises because the suppression of $Y_{B}$ in the presence of light $\tilde{b}_{R}$ and $\tilde{\tau}_{R}$ grows with $\tan \beta$. For the relatively flat portion of the green curves at lower $\tan \beta$, the suppression due to the $\tilde{b}_{R}$ and $\tilde{\tau}_{R}$ essentially compensates for the monotonic increase that would otherwise occur when these sfermions are heavy (the black curves). Eventually, the light RH sfermion suppression takes over, pushing the value of $Y_{B}$ below the 


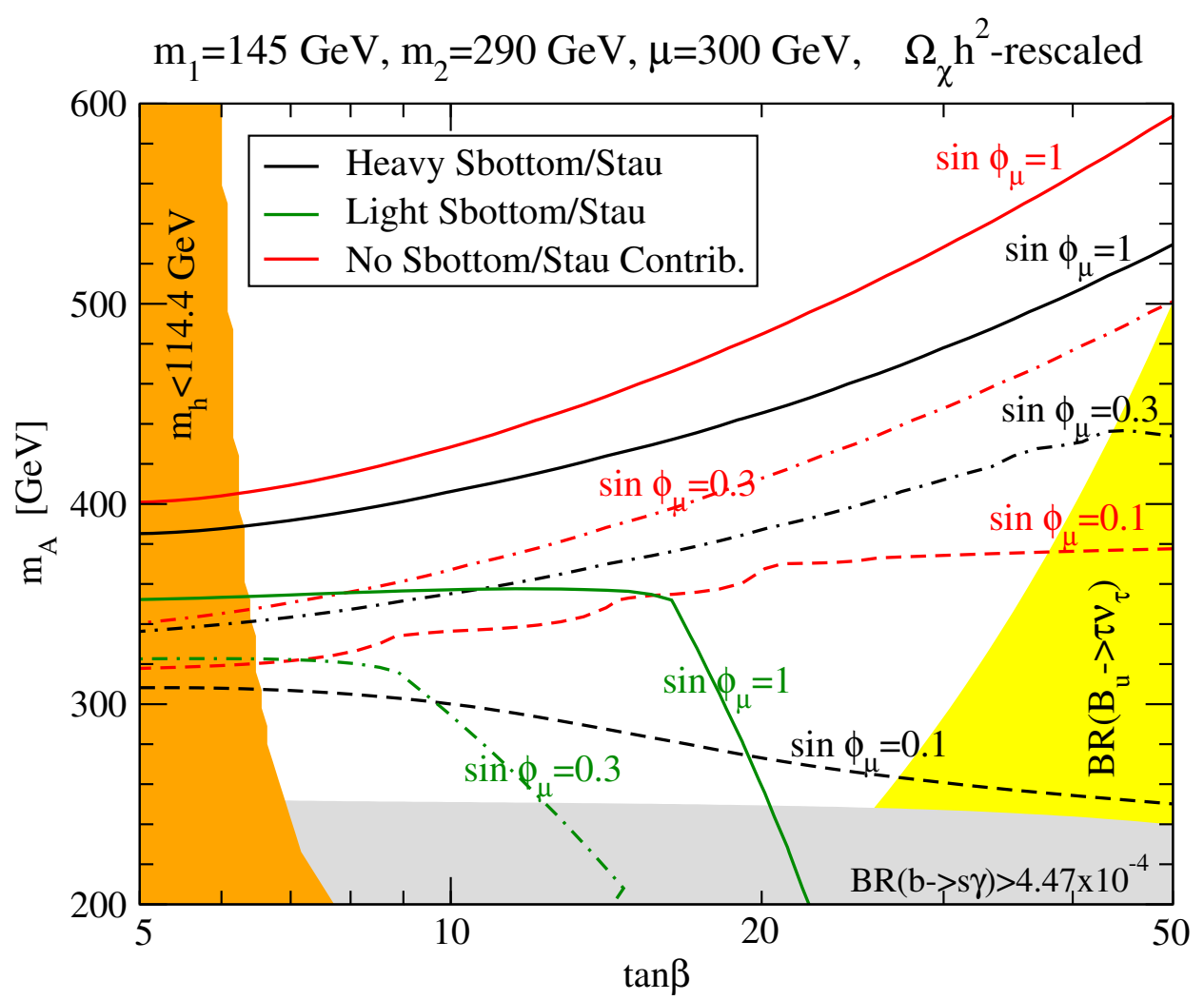

Figure 8. The same plane as in figure 7, but taking into account the effect of relic neutralino dilution in models over-producing dark matter on the CPV phases needed to achieve successful electroweak baryogenesis. The color code is the same as in figure 7 . We indicate with different colors lines corresponding to different assumptions on the mass of the Sbottom and of the Stau sfermions. Namely, our default assumption of heavy sfermions is indicated in black, while light $(300 \mathrm{GeV})$ sfermions correspond to the green line. Neglecting the effect of the Yukawa interactions involving sbottoms and staus produces the lines indicated in red.

experimental value and necessitating a smaller value of $m_{A}$ as needed to enhance the CPV source in the transport equations. This transition point corresponds to the elbow in a given isolevel curve. Interestingly, the resulting parameter space allowed by the requirement of successful EWB for $300 \mathrm{GeV}$ sfermions is now bounded, with $\tan \beta \lesssim 20$ and $m_{A} \lesssim 350 \mathrm{GeV}$. Note that we have not yet imposed EDM constraints.

We now investigate the size of the permanent EDMs of the electron (figure 9) and of the neutron (figure 10) over the same parameter space, taking into account the relic density rescaling, but still assuming super-heavy third generation sfermions. Our results are thus conservative here, given that including the effect of lighter sfermions would suppress $Y_{B}$ and force larger $\mathrm{CP}$ violating phases.

The shape of the contours in the figures illustrates how the size of the two-loop EDMs depends on both the size of $m_{A}$ (larger values suppress the two loop contributions where the heavy Higgs sector enters) and on $\tan \beta$. In addition, the EDMs also linearly depend on 


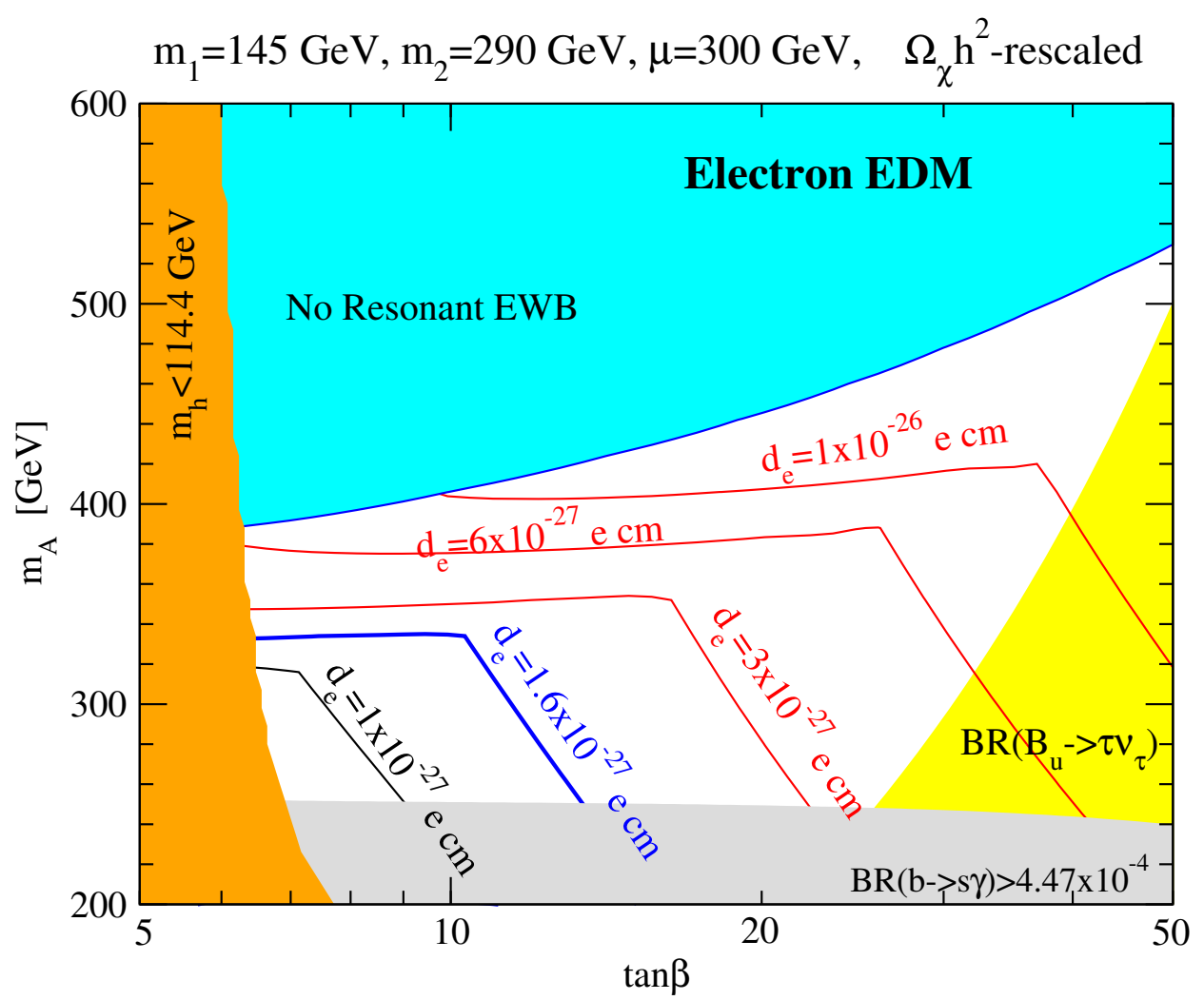

Figure 9. Contours of constant values for the two-loop electron EDM, on the same plane as the one shown in figure 8 (i.e including entropy rescaling). The blue line indicates the current experimental limit: the viable parameter space lies below that curve.

the size of the $\mathrm{CP}$ violating phases (that partly compensate for the suppression induced by larger values of $m_{A}$ ). As a result, the parameter space is bounded, and there is an absolute lower limit to the size of the EDMs on this slice of parameter space as well. Specifically, for the choice of parameters we make here, the electron EDM exceeds the current experimental limit for $m_{A} \gtrsim 330 \mathrm{GeV}$ and $\tan \beta \gtrsim 15$, with even more stringent constraints from the neutron EDM. The smallest the electron EDM can get on this slice of parameter space is a few $\times 10^{-28} \mathrm{e} \mathrm{cm}$, less than one order of magnitude below the current experimental sensitivity. The neutron EDM (figure 10) can at most be a factor of a few below the current sensitivity.

In short, the study of the $\left(\tan \beta, m_{A}\right)$ parameter space enforces the conclusion we obtained from the study of the orthogonal gaugino-higgsino parameter space: the size of the electron and of the neutron EDM, even only at the two-loop level, is bounded from below, and EWB will be thoroughly tested in the next generation of EDM search experiments. For the case of non-universal phases, we expect the EDM constraints on the $\left(\tan \beta, m_{A}\right)$ plane to be weaker, given the reduced sensitivity of EDMs to the bino phase (assuming it is the one responsible for resonant EWB). With an improvement of $O(100)$ in EDM sensitivity, however, we expect the restrictions on the parameter space in this plane will be similar to those shown for the universal phase case. 


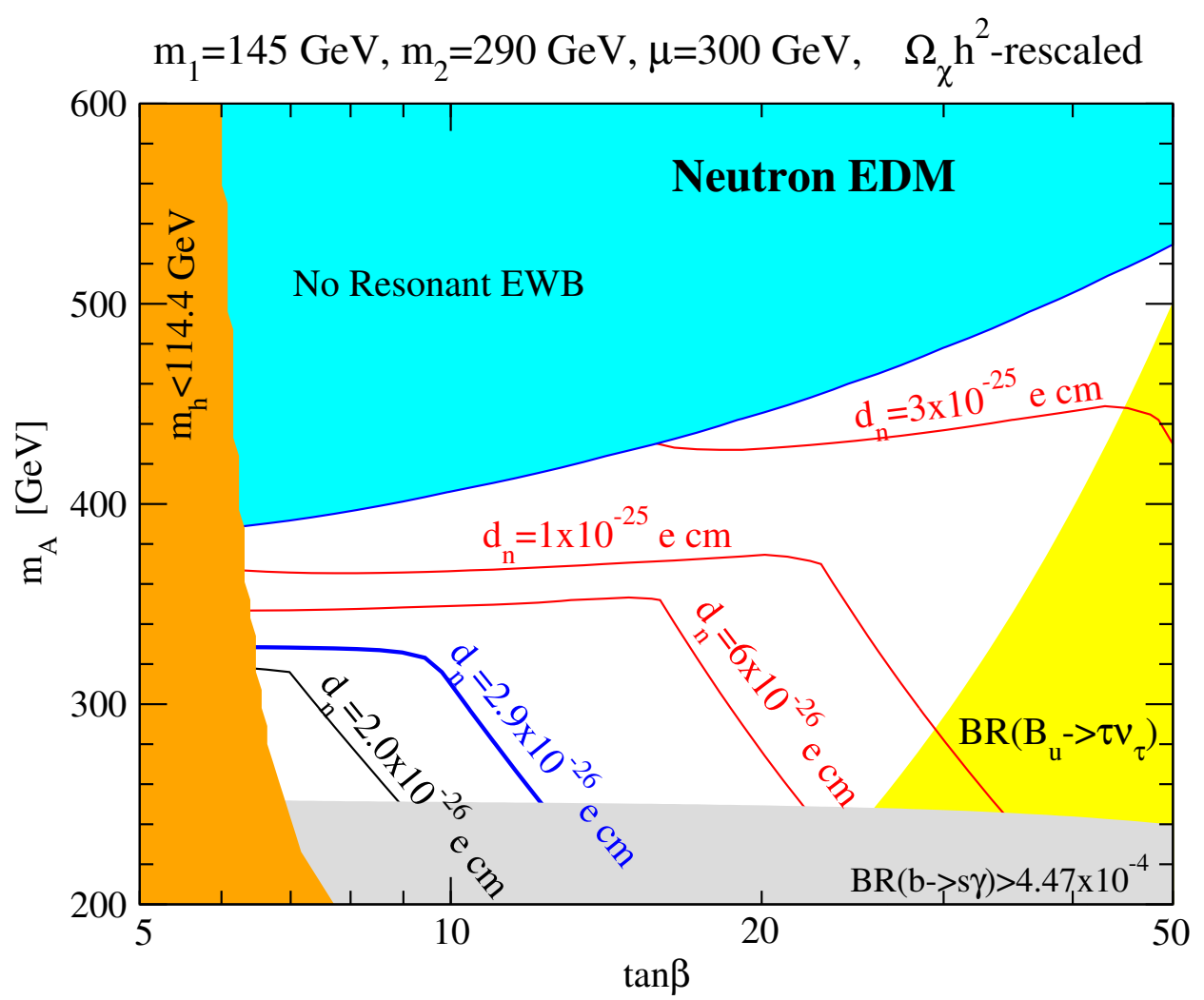

Figure 10. As in figure 9, this time for the two-loop neutron electric dipole moment.

\section{Discussion and conclusions}

In this paper we continued our program of investigating the phenomenology of supersymmetric models with successful electroweak baryogenesis. Our primary findings are:

1. Regions of parameter space with over abundant thermal relic neutralino production are either cosmologically ruled out or must occur in conjunction with a late-time reheating episode. In the latter instance, the consequent dilution of both the neutralino relic density and baryon number asymmetry implies that the baryon number density at the EWPT must have been larger. These considerations lead to more demanding requirements on the MSSM parameters in the domain of resonant winoHiggsino driven baryogenesis. As a result, we find that the parameter space for resonant wino-Higgsino driven baryogenesis is significantly more constrained than previously realized. In contrast, the requirements for successful bino-Higgsino driven baryogenesis are largely unaffected.

2. We explored for the first time the parameter space defined by the pair $m_{A}$ and $\tan \beta$. We find that a general upper limit exists to the maximal value the heavy Higgs mass scale can take, and this limit is even stronger if relic density dilution effects are taken into account. If third generation sfermions have a low mass scale, then an upper 
limit to $\tan \beta$ also emerges due role played by the third generation (s)fermions in the transport dynamics.

3. General lower limits exist on both the electron and the neutron EDMs, which are even stronger when the dilution effects are taken into account. Assuming phase universality, these lower bounds lie below the current experimental bounds by less than one order of magnitude. The non-observation of electron and neutron EDMs in experiments having ten times better sensitivity would likely rule out MSSM baryogenesis under the assumption of phase universality. These prospects can be seen from both the accessible regions of the $\left(\mu, M_{1,2}\right)$ parameter space and in the $\left(\tan \beta, M_{A}\right)$ plane.

4. If one relaxes the assumption of phase universality, then the expected lower bounds on $\left|d_{e}\right|$ and $\left|d_{n}\right|$ consistent with the scenario of resonant, bino-driven MSSM baryogenesis are roughly ten times smaller than we find under the assumption of phase universality. Because the strength of the CP-violating sources used in our analysis is likely to overpredict the strength of these sources that would likely result from a fully consistent, all-orders Higgs vev resummation, we expect that the resonant bino-driven scenario will be testable with the next generation electron and neutron EDM searches that aim for two orders of magnitude improved sensitivity.

The general picture that emerges from our present phenomenological update is one where MSSM electroweak baryogenesis is a predictive framework that will be conclusively tested in the near future. Indeed, the parameter space we combed in this analysis is devoid of regions where the predictions for specifically EDMs are unobservably small. Thus, the planned improvements in experimental sensitivity will put the entire setup under thorough scrutiny. Although there remain important open theoretical questions pertaining to the transport dynamics, such as the $\tan \beta$-dependence of the bubble wall profiles and consequences of a consistent resummation of the background field for the CP-violating sources, we expect that our general conclusions regarding the testability of MSSM baryogenesis will continue to hold after these issues are conclusively addressed.

\section{Acknowledgments}

We thank Sean Tulin for discussions and for providing help with part of the numerical results. The work of VC is supported by the Nuclear Physics Office of the U.S. Department of Energy under Contract No. DE-AC52- 06NA25396 and by the LDRD program at Los Alamos National Laboratory. MJRM and YL were supported in part by U.S. Department of Energy contract DE-FG02-08ER41531 and by the Wisconsin Alumni Research Foundation. MJRM also thanks the Aspen Center for Physics and TRIUMF Theory Group where part of this work was completed. S.P. is partly supported by an Outstanding Junior Investigator Award from the U.S. Department of Energy, Office of Science, High Energy Physics, DoE Contract DEFG02-04ER41268, and by NSF Grant PHY-0757911. 
Open Access. This article is distributed under the terms of the Creative Commons Attribution Noncommercial License which permits any noncommercial use, distribution, and reproduction in any medium, provided the original author(s) and source are credited.

\section{References}

[1] Particle Data Group collaboration, W.M. Yao et al., Review of particle physics, J. Phys. G 33 (2006) 1 [SPIRES].

[2] WMAP collaboration, J. Dunkley et al., Five-year Wilkinson Microwave Anisotropy Probe (WMAP) observations: likelihoods and parameters from the WMAP data, Astrophys. J. Suppl. 180 (2009) 306 [arXiv:0803.0586] [SPIRES].

[3] A.D. Sakharov, Violation of CP invariance, $C$ asymmetry and baryon asymmetry of the universe, Pisma Zh. Eksp. Teor. Fiz. 5 (1967) 32 [JETP Lett. 5 (1967) 24] [Sov. Phys. Usp. 34 (1991) 392] [SPIRES].

[4] LeP Working Group for Higgs boson searches collaboration, R. Barate et al., Search for the standard model Higgs boson at LEP, Phys. Lett. B 565 (2003) 61 [hep-ex/0306033] [SPIRES].

[5] G.R. Farrar and M.E. Shaposhnikov, Baryon asymmetry of the universe in the standard electroweak theory, Phys. Rev. D 50 (1994) 774 [hep-ph/9305275] [SPIRES].

[6] A.I. Bochkarev and M.E. Shaposhnikov, Electroweak production of baryon asymmetry and upper bounds on the Higgs and top masses, Mod. Phys. Lett. A 2 (1987) 417 [SPIRES].

[7] H. Baer and X. Tata, Weak scale supersymmetry, Cambridge University Press, Cambridge U.K. (2006) [ISBN-10:0521857864] [ISBN-13:9780521857864] [SPIRES].

[8] W.C. Griffith et al., Improved limit on the permanent electric dipole moment of ${ }^{199} \mathrm{Hg}$, Phys. Rev. Lett. 102 (2009) 101601 [SPIRES].

[9] M. Carena, G. Nardini, M. Quirós and C.E.M. Wagner, The effective theory of the light stop scenario, JHEP 10 (2008) 062 [arXiv:0806.4297] [SPIRES].

[10] Y. Li, S. Profumo and M. Ramsey-Musolf, Higgs-higgsino-gaugino induced two loop electric dipole moments, Phys. Rev. D 78 (2008) 075009 [arXiv:0806.2693] [SPIRES].

[11] Y. Li, S. Profumo and M. Ramsey-Musolf, Bino-driven electroweak baryogenesis with highly suppressed electric dipole moments, Phys. Lett. B 673 (2009) 95 [arXiv:0811.1987] [SPIRES].

[12] D.J.H. Chung, B. Garbrecht, M.J. Ramsey-Musolf and S. Tulin, Supergauge interactions and electroweak baryogenesis, arXiv:0908.2187 [SPIRES].

[13] D.J.H. Chung, B. Garbrecht, M.J. Ramsey-Musolf and S. Tulin, Lepton-mediated electroweak baryogenesis, arXiv:0905.4509 [SPIRES].

[14] M. Carena, G. Nardini, M. Quirós and C.E.M. Wagner, The baryogenesis window in the MSSM, Nucl. Phys. B 812 (2009) 243 [arXiv: 0809.3760] [SPIRES].

[15] C. Lee, V. Cirigliano and M.J. Ramsey-Musolf, Resonant relaxation in electroweak baryogenesis, Phys. Rev. D 71 (2005) 075010 [hep-ph/0412354] [SPIRES].

[16] V. Cirigliano, M.J. Ramsey-Musolf, S. Tulin and C. Lee, Yukawa and tri-scalar processes in electroweak baryogenesis, Phys. Rev. D 73 (2006) 115009 [hep-ph/0603058] [SPIRES]. 
[17] D.J.H. Chung, B. Garbrecht, M.J. Ramsey-Musolf and S. Tulin, Yukawa interactions and supersymmetric electroweak baryogenesis, Phys. Rev. Lett. 102 (2009) 061301 [arXiv:0808.1144] [SPIRES].

[18] M.S. Carena, M. Quirós, M. Seco and C.E.M. Wagner, Improved results in supersymmetric electroweak baryogenesis, Nucl. Phys. B 650 (2003) 24 [hep-ph/0208043] [SPIRES].

[19] T. Konstandin, T. Prokopec and M.G. Schmidt, Kinetic description of fermion flavor mixing and CP-violating sources for baryogenesis, Nucl. Phys. B 716 (2005) 373 [hep-ph/0410135] [SPIRES].

[20] T. Konstandin, T. Prokopec and M.G. Schmidt, Axial currents from CKM matrix CP-violation and electroweak baryogenesis, Nucl. Phys. B 679 (2004) 246 [hep-ph/0309291] [SPIRES].

[21] J.M. Moreno, M. Quirós and M. Seco, Bubbles in the supersymmetric standard model, Nucl. Phys. B 526 (1998) 489 [hep-ph/9801272] [SPIRES].

[22] M. Pietroni, The electroweak phase transition in a nonminimal supersymmetric model, Nucl. Phys. B 402 (1993) 27 [hep-ph/9207227] [SPIRES].

[23] S. Profumo, M.J. Ramsey-Musolf and G. Shaughnessy, Singlet Higgs phenomenology and the electroweak phase transition, JHEP 08 (2007) 010 [arXiv:0705.2425] [SPIRES].

[24] R. Fok and G.D. Kribs, Four generations, the electroweak phase transition and supersymmetry, Phys. Rev. D 78 (2008) 075023 [arXiv:0803.4207] [SPIRES].

[25] J. Shu, T.M.P. Tait and C.E.M. Wagner, Baryogenesis from an earlier phase transition, Phys. Rev. D 75 (2007) 063510 [hep-ph/0610375] [SPIRES].

[26] H. Patel and M.J. Ramsey-Musolf, in preparation.

[27] P. Fileviez Perez, T. Han, G.-Y. Huang, T. Li and K. Wang, Neutrino masses and the LHC: testing type II seesaw, Phys. Rev. D 78 (2008) 015018 [arXiv:0805.3536] [SPIRES].

[28] J. Kehayias and S. Profumo, Semi-analytic calculation of the gravitational wave signal from the electroweak phase transition for general quartic scalar effective potentials, arXiv:0911.0687 [SPIRES].

[29] D.H. Lyth and E.D. Stewart, Thermal inflation and the moduli problem, Phys. Rev. D 53 (1996) 1784 [hep-ph/9510204] [SPIRES].

[30] L. Knox and M.S. Turner, Inflation at the electroweak scale, Phys. Rev. Lett. 70 (1993) 371 [astro-ph/9209006] [SPIRES].

[31] G. Nardini, M. Quirós and A. Wulzer, A confining strong first-order electroweak phase transition, JHEP 09 (2007) 077 [arXiv:0706.3388] [SPIRES].

[32] M. Pospelov, Particle physics catalysis of thermal big bang nucleosynthesis, Phys. Rev. Lett. 98 (2007) 231301 [hep-ph/0605215] [SPIRES].

[33] V. Cirigliano, S. Profumo and M.J. Ramsey-Musolf, Baryogenesis, electric dipole moments and dark matter in the MSSM, JHEP 07 (2006) 002 [hep-ph/0603246] [SPIRES].

[34] C. Wainwright and S. Profumo, The impact of a strongly first-order phase transition on the abundance of thermal relics, Phys. Rev. D 80 (2009) 103517 [arXiv:0909.1317] [SPIRES].

[35] D. Eriksson, F. Mahmoudi and O. Stal, Charged Higgs bosons in minimal supersymmetry: updated constraints and experimental prospects, JHEP 11 (2008) 035 [arXiv:0808.3551] [SPIRES].

[36] M. Antonelli et al., Flavor physics in the quark sector, arXiv:0907.5386 [SPIRES]. 